\title{
Retention in care in aging adults with a dual diagnosis of HIV infection and type 2 diabetes mellitus: a longitudinal retrospective cross-sectional study
}

\author{
Julie Ann Zuniga ${ }^{1 \dagger}$, Alexandra A. García ${ }^{1,2}$, Junse Lee ${ }^{3}$ and Jungmin Park ${ }^{4^{*}}$ (D)
}

\begin{abstract}
Background: This study aimed to investigate the measures of retention in care (RIC) in persons living with HIV (PLWH) and type 2 diabetes mellitus (T2DM) by age group (younger vs. older adults).

Methods: This was a longitudinal retrospective cross-sectional study that used secondary data from the Center for AIDS Research Network of Integrated Clinical Systems (CNICS). We examined RIC in 798 adult PLWH + T2DM who visited a CNICS clinic at least once in 2015. Six measures of RIC were examined: missed visits [measured as a continuous variable (total number of missed visits) and dichotomous variable $(0=$ never missed, $1=$ missed)], visit adherence, 6-month visit gap, 4-month visit constancy, and the Health and Resources Services Administration HIV/AIDS Bureau's $\mathrm{RIC}$ measure. We calculated Spearman correlation coefficients and conducted logistic regression and multi-group path analysis.
\end{abstract}

Results: Most RIC measures were significantly correlated $(p<0.05)$ with one another; only 4-month visit constancy was not correlated with other measures. Except for the number of missed visits in older adult PLWH+T2DM, we found no significant relationships between RIC measures and CD4 cell count using logistic regression. However, multi-group path analysis demonstrated significant positive relationships between most RIC measures and CD4 cell count in both age groups. In younger adults living with HIV (YALWH) +T2DM, HbA1c level, but not CD4 count, was significantly associated with most RIC measures.

Conclusions: $\mathrm{RIC}$ is related to disease control (CD4 cell count and HbA1c level) in PLWH+T2DM and notably, HbA1c level was only significantly affected in YALWH + T2DM. A future study is needed to find more accurate reasons for the fact that only HbA1c level had significant relationships in YALWH+T2DM. The findings from this study provide guidance in measuring RIC in PLWH who have comorbidities.

Keywords: CD4 count, Type 2 diabetes mellitus, HIV, Retention in care, Comorbidity

*Correspondence: jminpark@hanyang.ac.kr

†Julie Ann Zuniga_-First author

${ }^{4}$ School of Nursing, Hanyang University, 222 Wangsimni-ro,

Sageun-dong, Seongdong-gu, Seoul, South Korea

Full list of author information is available at the end of the article

\section{Background}

Presently, life expectancy for $>1.2$ million persons living with HIV (PLWH) is comparable to that for the general population $[1,2]$, in part because of the development of effective antiretroviral therapy (ART) [3]. However, as PLWH age, more than half develop at least one comorbidity [4]. In older adults (aged $\geq 50$ years)

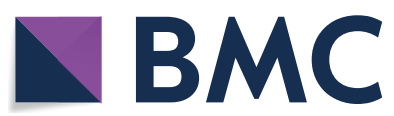

(c) The Author(s) 2020. This article is licensed under a Creative Commons Attribution 4.0 International License, which permits use, sharing, adaptation, distribution and reproduction in any medium or format, as long as you give appropriate credit to the original author(s) and the source, provide a link to the Creative Commons licence, and indicate if changes were made. The images or other third party material in this article are included in the article's Creative Commons licence, unless indicated otherwise in a credit line to the material. If material is not included in the article's Creative Commons licence and your intended use is not permitted by statutory regulation or exceeds the permitted use, you will need to obtain permission directly from the copyright holder. To view a copy of this licence, visit http://creativeco mmons.org/licenses/by/4.0/. The Creative Commons Public Domain Dedication waiver (http://creativecommons.org/publicdomain/ zero/1.0/) applies to the data made available in this article, unless otherwise stated in a credit line to the data. 
living with HIV (OALWH), type 2 diabetes mellitus (T2DM) is one of the most common comorbidities [5-8]. Approximately $10-15 \%$ of PLWH have T2DM, a prevalence rate slightly higher than that in persons without HIV [6, 9, 10]. Because the prevalence of T2DM increases with age and the population of older adults with T2DM is expected to increase in the future [11], the same trend is also likely to be reflected in the PLWH population.

In 2014, OALWH accounted for $17 \%$ of PLWH, and the proportion is growing [12-17]. Because OALWH have more comorbidities than younger adults with HIV (YALWH, aged 19-49 years), OALWH encounter unique barriers to HIV management [18]. Approximately $30 \%$ of OALWH have at least two chronic conditions [19], and controlling both HIV infection and T2DM is the key to survival, complication prevention, and maintenance of quality of life. To achieve longterm control of HIV infection, PLWH must visit their healthcare providers at regular intervals, an important predictor of health called retention in care (RIC). RIC is important in successful HIV management because it enables the monitoring of combination ART, evaluation of medication toxicity, and identification of HIV treatment failure [20]. Additionally, RIC has been associated with the ultimate goal of HIV care, i.e., undetectable HIV viral load [21]. Undetectable viral load greatly decreases the risk of HIV transmission [22].

It is recommended that PLWH visit their healthcare provider every 3-6 months for blood tests so that their condition can be monitored. Once the virus is stable and undetectable, PLWH may decrease the number of annual visits [23]. Approximately 51\% of PLWH are retained in care after diagnosis [24]. However, even though PLWH retained in care can expect improved survival rates [25] and RIC has been associated with positive HIV outcomes, it is unknown whether RIC is also associated with positive outcomes in those with comorbidities, such as T2DM.

RIC can be measured using several different methods including missed appointments, number of appointments, and number of appointments within a certain time frame [26]. However, not all RIC measures accurately reflect health benefits and risks, depending on patients' disease progression, ART initiation, and other medical conditions that may need close monitoring, such as T2DM. This study aimed to investigate the relationships of RIC measures in PLWH + T2DM in two age groups (older vs. younger adults) and explore the relationships between RIC and health outcomes relevant to HIV infection and T2DM.

\section{Methods}

\section{Research design}

This was a longitudinal retrospective cross-sectional study that used secondary analysis of data from the Center for AIDS Research (CFAR) Network of Integrated Clinic Systems (CNICS), which includes medical records of PLWH from 1995. The longitudinal retrospective cross-sectional study design was based on the study by Mugavero et al. [26] which set the "gold standard" of measurements in PLWH regardless of comorbidities. This study was exempt from review by the Institutional Review Board of the University of Texas at Austin because the data were already de-identified.

\section{Measures}

The CNICS database contains medical records of $>32,000$ patients from eight CFAR clinics throughout the USA, including 798 PLWH + T2DM aged $>18$ years who received ART from CNICS clinics for at least 6 months and had at least one primary care appointment at a CNICS clinic in 2015. Diabetes diagnosis was verified by $\mathrm{HbA} 1 \mathrm{c}$ levels $>6.5 \%$ or use of medications for T2DM. The dataset excluded patients with type 1 diabetes because our focus was on PLWH + T2DM. Data included age ( $\geq 50$ years old, $<50$ years old), sex, race/ethnicity, number of clinic visits in 2015, and CD4 count, which was analyzed at CNICS clinics. Three groups were created based on the CD4 count: $\geq 500$ cells $/ \mu \mathrm{L}, 200-499$ cells $/ \mu \mathrm{L}$, and $<200$ cells $/ \mu \mathrm{L}$ [27]. Six RIC measures were used: missed clinic visits (count, dichotomous), visit adherence, 4-month visit constancy, 6-month visit gap, and the US Health Resources and Services Administration HIV/AIDS Bureau (HRSA HAB) measure.

- Missed visits were unkept appointments that were not cancelled by the patient or clinic staff. Missed visits were captured continuously (summed for the total number of missed visits) and dichotomously $(0=$ kept the visit, $1=$ missed the visit). More missed visits indicate worse RIC.

- Visit adherence is a continuously measured variable of the proportion of appointments kept versus that of the appointments scheduled. More kept visits show better RIC.

- Four-month visit constancy is the sum of 4-month intervals with $\geq$ one kept visit (range, $0-3$ ).

- Six-month visit gap is the number of episodes with $\geq 189$ days between kept visits $[0=$ not retained, $1=$ retained ( $\geq$ one visit in 6 months)].

- HRSA HAB measure (90-day gap) is attendance at visits $\geq 90$ days apart $[0=$ not retained, 
$1=$ retained (two kept visits separated by $\geq 90$ days in a 12-month period)] [21].

For health outcome variables, we obtained two outcome variables for PLWH + T2DM representing HIV and T2DM control: CD4 count at the last visit and most recent $\mathrm{HbA1c}$ level, respectively. A CD4 count $<200$ cells $/ \mu \mathrm{L}$ indicates the last stage of HIV infection. In AIDS, a CD4 count $>500$ cells $/ \mu \mathrm{L}$ represents a normal value, and a count between 200 cells/ $\mu \mathrm{L}$ and 500 cells $/ \mu \mathrm{L}$ indicates HIV treatment with sustained good health status [27]. HbA1c level reflects the level of glycemia for 3 months [28], an indicator of T2DM management [29]. T2DM control in PLWH is represented by HbA1c levels $<6.5 \%[30,31]$.

\section{Statistical analysis}

Descriptive statistics using means, standard deviations, ranges of scores, frequencies, and percentages were applied to analyze demographic characteristics [age, sex, race/ethnicity, missed visits (count, dichotomous), attendance, visit adherence, 4-month visit constancy, 6-month visit gap, HRSA HAB measure, HbA1c level, and CD4 count]. Spearman's correlation coefficients were used to evaluate associations among six RIC measures [missed visits (count, dichotomous), visit adherence, 4-month visit constancy, 6-month visit gap, HRSA HAB measure], health outcomes (CD4 count, HbA1c level), and age groups ( $\geq 50$ years, $<50$ years). Logistic regression was used to evaluate RIC measures and age groups as predictors of HIV status determined by CD4 count. Logistic regression was also used to predict the symptom burden in PLWH + T2DM (as measured by the HIV symptom index) using age group and RIC measure [using the HRSA HAB measure (not retained vs. retained 90-day gap)]. Finally, to investigate the relationships between RIC measure and disease control (CD4 count, HbA1c level) by age group, we conducted multi-group path analysis. In the path analysis, missed visits (count), visit attendance (count, actual visit clinic times), visit adherence, and 4-month visit constancy for RIC were considered independent variables, and the CD4 count and $\mathrm{HbA1c}$ level were considered dependent variables. The Chi square, normed fit index (NFI), Tucker-Lewis Index (TLI), comparative fit index (CFI), and root mean square error of approximation (RMSEA) were used to estimate model fit. Statistical significance in all tests was set at $\alpha=0.05$. The statistical analyses were conducted using IBM SPSS version 23.0 [32] and AMOS version 23.0 [33].

\section{Results}

All patients in this study $(N=798)$ were PLWH + T2DM. They were predominantly male $(77 \%)$, aged 55 years, and fairly racially and ethnically diverse ( $41 \%$ White nonHispanic, 40\% African American non-Hispanic, 18\% Hispanic). They had fairly well-controlled conditions (mean HbA1c level $=7.6 \%$; mean CD4 count $=718$ ) and were retained in care (missed visits days average: 0.29 day) with an average of three visits in 2015 (Table 1).

Older adults ( $>50$ years, $n=564)$ had a mean age of 59 years, and younger adults $(n=234)$ were approximately 15 years younger, with a mean age of 45 years. The two groups were statistically similar in almost all RIC measures and CD4 count and HbA1c level $(p>0.05)$. However, there were significant differences in sex, race/ ethnicity, and attendance $(p<0.05)$ between the older and younger adults. There were more older men $(80 \%)$ than younger men $(71 \% ; p=0.01)$ in the sample and more African American non-Hispanic patients among younger adults (45.3\%; $p=0.001)$ than among older adults $(40.3 \%$; Table 1).

\section{RIC}

To evaluate the relationships among the six RIC measures in PLWH + T2DM, we conducted Spearman's correlations (Table 2). Dichotomously measured missed visits were highly positively correlated with continuously measured missed visits $(r=0.996, p<0.01)$. Both measures of missed visits (count, dichotomous) were highly negatively correlated with visit adherence $(r=-0.854, p<0.01)$. However, 4-month visit constancy and 6-month visit gap had a minimally negative correlation $(r=-0.105, p<0.01)$, but neither demonstrated significant correlations with missed visits (either count or dichotomous) or visit adherence. The HRSA HAB measure was minimally positively correlated with visit adherence $(r=0.130, p<0.01)$ and 6-month visit gap $(r=0.169$, $p<0.01)$ and negatively correlated with missed visits (both count and dichotomous) ( $r=-0.141$ and -0.140 , respectively, $p<0.01)$. The HRSA HAB measure had a high positive correlation with 4-month visit constancy $(r=0.93, p<0.05)$.

\section{$O A L W H+T 2 D M$}

Spearman's correlations for all RIC measures in older adults showed almost the same pattern as those in the whole sample of PLWH (Table 3). As in the whole sample, missed visits (count and dichotomous) had a highly positively correlation and were highly negatively correlated with visit adherence. Similarly, 4-month visit constancy and 6-month visit gap had a significant association but were not significantly correlated 
Table 1 PLWH + T2DM characteristics

\begin{tabular}{|c|c|c|c|c|}
\hline Characteristics & $\begin{array}{l}\text { PLWH+T2DM } \\
\text { All age groups }(N=798)\end{array}$ & OALWH +T2DM $(n=564)$ & YALWH + T2DM $(n=234)$ & $\begin{array}{l}\text { Pearson Chi Square or Fisher } \\
\text { Exact }{ }^{\mathrm{a}}(\mathrm{OALWH}+\mathrm{T} 2 \mathrm{DM} \text {, } \\
\text { YALWH+T2DM) }\end{array}$ \\
\hline Age (years) mean $\pm S D$ & $55.01 \pm 8.96$ & $59.35 \pm 7.22(70.7 \%)$ & $44.56 \pm 5.10(29.3 \%)$ & \\
\hline Gender & & & & $0.01^{* *}$ \\
\hline Male & $618(77.4 \%)$ & $451(80 \%)$ & $167(71.4 \%)$ & \\
\hline Female & $180(22.6 \%)$ & $113(20 \%)$ & $67(28.6 \%)$ & \\
\hline Race/ethnicity & $N=794$ & $N=560$ & & $0.001^{* * *}$ \\
\hline White-non-Hispanic & $326(41.1 \%)$ & $253(45.2 \%)$ & $73(31.2 \%)$ & \\
\hline White-Hispanic & $98(12.3 \%)$ & $62(11.1 \%)$ & $36(15.4 \%)$ & \\
\hline $\begin{array}{l}\text { African American-non- } \\
\text { Hispanic }\end{array}$ & $320(40.3 \%)$ & $214(38.2 \%)$ & $106(45.3 \%)$ & \\
\hline African American-Hispanic & $3(0.4 \%)$ & $0(0 \%)$ & $3(1.3 \%)$ & \\
\hline Hispanic & $18(2.3 \%)$ & $12(2.1 \%)$ & $6(2.6 \%)$ & \\
\hline Others & $29(3.7 \%)$ & $19(3.4 \%)$ & $10(4.3 \%)$ & \\
\hline Missed visits (count) & Range: 0-12 & Range: $0-5$ & Range: 0-12 & 0.23 \\
\hline Mean $\pm S D$ & $0.29 \pm 0.90$ & $0.24 \pm 0.66$ & $0.41 \pm 1.30$ & \\
\hline Zero & $667(83.6 \%)$ & $476(84.4 \%)$ & $191(81.6 \%)$ & \\
\hline One & $84(10.5 \%)$ & $61(10.8 \%)$ & $23(9.8 \%)$ & \\
\hline Two & $27(3.4 \%)$ & $16(2.8 \%)$ & $11(4.7 \%)$ & \\
\hline$\geq$ Three & $20(2.4 \%)$ & $11(2 \%)$ & $9(3.7 \%)$ & \\
\hline Missed visits (dichotomous) & & & & 0.35 \\
\hline No missed visits & $667(83.6 \%)$ & $476(84.4 \%)$ & $191(81.6 \%)$ & \\
\hline At least one missed visit & $1(16.4 \%)$ & $88(15.6 \%)$ & $43(18.4 \%)$ & \\
\hline Visit attendance & Range: $0-26$ days & Range: $0-26$ days & Range: $0-26$ days & $0.01^{* *}$ \\
\hline Mean \pm SD & $3.07 \pm 2.517$ & $3.05 \pm 2.417$ & $3.12 \pm 2.747$ & \\
\hline Visit adherence & & & & 0.06 \\
\hline Mean \pm SD & $0.904 \pm 0.24$ & $0.910 \pm 0.23$ & $0.890 \pm 0.25$ & \\
\hline $0-24 \%$ & $33(4.1 \%)$ & $23(4.1 \%)$ & $10(4.3 \%)$ & \\
\hline $25-49 \%$ & $15(1.8 \%)$ & $12(2.1 \%)$ & $3(1.3 \%)$ & \\
\hline $50-74 \%$ & $62(7.9 \%)$ & $34(6 \%)$ & $28(11.6 \%)$ & \\
\hline $75-99 \%$ & $47(5.9 \%)$ & $37(6.6 \%)$ & $10(4.2 \%)$ & \\
\hline $100 \%$ & $641(80.3 \%)$ & $458(81.2 \%)$ & $183(78.2 \%)$ & \\
\hline $\begin{array}{l}\text { 4-month visit constancy (inter- } \\
\text { vals with } \geq 1 \text { kept visit) }\end{array}$ & $n=772$ & $n=546$ & $n=226$ & 0.67 \\
\hline Zero & $7(0.9 \%)$ & $5(0.9 \%)$ & $2(0.9 \%)$ & \\
\hline One & $207(26.8 \%)$ & $143(26.2 \%)$ & $64(28.3 \%)$ & \\
\hline Two & $334(43.3 \%)$ & $244(44.7 \%)$ & $90(39.8 \%)$ & \\
\hline Three & $224(29 \%)$ & $154(28.2 \%)$ & $70(31 \%)$ & \\
\hline $\begin{array}{l}\text { 6-month gap ( } \geq 189 \text { days } \\
\text { between sequential kept } \\
\text { visits) }\end{array}$ & $n=606$ & $n=425$ & $n=181$ & 0.19 \\
\hline Retained & $570(94.1 \%)$ & $396(93.2 \%)$ & $174(96.1 \%)$ & \\
\hline Not retained & $36(5.9 \%)$ & $29(6.8 \%)$ & $7(3.9 \%)$ & \\
\hline $\begin{array}{l}\text { HRSA HAB measure ( } 2 \text { kept } \\
\text { visits }>90 \text { days apart) }\end{array}$ & $n=606$ & $n=425$ & $n=181$ & 0.39 \\
\hline Retained & $418(69 \%)$ & $298(70.1 \%)$ & $120(66.3 \%)$ & 0.07 \\
\hline Not retained & $188(31 \%)$ & $127(29.9 \%)$ & $61(33.7 \%)$ & \\
\hline HbA1c level & $n=179$ & $n=179$ & $n=179$ & 0.07 \\
\hline Mean \pm SD & $7.565 \pm 1.93$ & $7.529 \pm 1.90$ & $7.69 \pm 2.03$ & \\
\hline$\leq 6.5 \%$ & $64(35.8 \%)$ & $50(36 \%)$ & $14(35 \%)$ & \\
\hline$>6.5 \%$ & $115(64.2 \%)$ & $89(64 \%)$ & $26(65 \%)$ & \\
\hline
\end{tabular}


Table 1 (continued)

\begin{tabular}{|c|c|c|c|c|}
\hline Characteristics & $\begin{array}{l}\mathrm{PLWH}+\mathrm{T} 2 \mathrm{DM} \\
\text { All age groups }(N=798)\end{array}$ & OALWH + T2DM $(n=564)$ & YALWH + T2DM $(n=234)$ & $\begin{array}{l}\text { Pearson Chi Square or Fisher } \\
\text { Exact }^{\mathrm{a}}(\mathrm{OALWH}+\mathrm{T} 2 \mathrm{DM} \text {, } \\
\text { YALWH+T2DM) }\end{array}$ \\
\hline CD4 count & $n=194$ & $n=151$ & $n=43$ & 0.61 \\
\hline Mean $\pm S D$ & $717.84 \pm 407.42$ & $696.44 \pm 387.06$ & $792.98 \pm 469.35$ & \\
\hline$\geq 500$ cells $/ \mu \mathrm{L}$ & $132(68 \%)$ & $102(67.5 \%)$ & $30(69.8 \%)$ & \\
\hline 200-499 cells/MluL & $49(25.3 \%)$ & $40(26.5 \%)$ & $9(20.9 \%)$ & \\
\hline$<200$ cells $/ \mu \mathrm{L}$ & $13(6.7 \%)$ & $9(6 \%)$ & $4(9.3 \%)$ & \\
\hline
\end{tabular}

Some variables have different sample sizes

HRSA HAB measure the US Health Resources and Services Administration HIV/AIDS Bureau (HRSA HAB) measure, PLWH+T2DM persons living with HIV and diabetes, all ages, $O A L W H+T 2 D M$ older adults with HIV and T2DM, YALWH + T2DM younger adults with HIV and T2DM

${ }^{*} p<0.05,{ }^{* *} p<0.01,{ }^{* * *} p<0.00$

a Pearson Chi Square or Fisher Exact between OALWH+T2DM and YALWH+T2DM

Table 2 Spearman's correlation coefficients of RIC measures for PLWH + T2DM, all age groups

\begin{tabular}{|c|c|c|c|c|c|c|}
\hline & Missed visits (count) & $\begin{array}{l}\text { Missed visits } \\
\text { (dichotomous) }\end{array}$ & Visit adherence & $\begin{array}{l}\text { 4-month } \\
\text { visit } \\
\text { constancy }\end{array}$ & 6-month gap & $\begin{array}{l}\text { HRSA } \\
\text { HAB } \\
\text { measure }\end{array}$ \\
\hline Missed visits (count, range: $0-12$ ) & 1 & & & & & \\
\hline Missed visits (dichotomous) & $0.996^{* *}$ & 1 & & & & \\
\hline Visit adherence (continuous, range: $0-1$ ) & $-0.854^{* *}$ & $-0.854^{* *}$ & 1 & & & \\
\hline $\begin{array}{l}\text { 4-month visit constancy (categorical, range: } \\
0-3 \text { ) }\end{array}$ & 0.005 & 0 & 0.33 & 1 & & \\
\hline 6-month gap (dichotomous) & 0.02 & 0.024 & -0.029 & $-0.105^{* *}$ & 1 & \\
\hline HRSA HAB measure (dichotomous) & $-0.141^{* *}$ & $-0.140^{* *}$ & $0.130^{* *}$ & $0.93^{*}$ & $0.169^{* *}$ & 1 \\
\hline
\end{tabular}

Some variables have different sample sizes

HRSA HAB measure the US Health Resources and Services Administration HIV/AIDS Bureau (HRSA HAB) measure, PLWH + T2DM persons living with HIV and diabetes, all ages, OALWH + T2DM older adults with HIV and T2DM, YALWH + T2DM younger adults with HIV and T2DM

${ }^{*} p<0.05,{ }^{* *} p<0.01$

Table 3 Spearman's correlation coefficients of RIC measures for OALWH + T2DM

\begin{tabular}{|c|c|c|c|c|c|c|}
\hline & Missed visits (count) & $\begin{array}{l}\text { Missed visits } \\
\text { (dichotomous) }\end{array}$ & Visit adherence & $\begin{array}{l}\text { 4-month } \\
\text { visit } \\
\text { constancy }\end{array}$ & 6-month gap & $\begin{array}{l}\text { HRSA } \\
\text { HAB } \\
\text { measure }\end{array}$ \\
\hline Missed visits (count, range: $0-5$ ) & 1 & & & & & \\
\hline Missed visits (dichotomous) & $0.997^{* *}$ & 1 & & & & \\
\hline Visit adherence (continuous, range: 0-1.0) & $-0.854^{* *}$ & $-0.855^{* *}$ & 1 & & & \\
\hline $\begin{array}{l}\text { 4-month visit constancy (categorical, range: } \\
0-3 \text { ) }\end{array}$ & 0.022 & 0.023 & 0.11 & 1 & & \\
\hline 6-month gap (dichotomous) & -0.009 & -0.003 & -0.002 & $-0.126^{* *}$ & 1 & \\
\hline HRSA HAB measure (dichotomous) & $-0.143^{* *}$ & $-0.143^{* *}$ & $0.130^{* *}$ & 0.05 & $0.177^{* *}$ & 1 \\
\hline
\end{tabular}

HRSA HAB measure, the US Health Resources and Services Administration HIV/AIDS Bureau (HRSA HAB) measure, OALWH+T2DM older adults with HIV and T2DM ** $p<0.01$

with missed visits (count, dichotomous) or visit adherence. Unlike that in PLWH + T2DM, the HRSA HAB measure in OALWH + T2DM was not correlated with 4-month visit constancy.
YALWH + T2DM

The pattern of correlations for YALWH + T2DM differed from those for PLWH + T2DM and OALWH + T2DM (Table 4). Most notably, the HRSA 
Table 4 Spearman's correlation coefficients of RIC measures for YALWH+T2DM

\begin{tabular}{|c|c|c|c|c|c|c|}
\hline & Missed visits (count) & $\begin{array}{l}\text { Missed visits } \\
\text { (dichotomous) }\end{array}$ & Visit adherence & $\begin{array}{l}\text { 4-month } \\
\text { visit } \\
\text { constancy }\end{array}$ & 6-month gap & $\begin{array}{l}\text { HRSA } \\
\text { HAB } \\
\text { measure }\end{array}$ \\
\hline Missed visits (count, range: 0-12) & 1 & & & & & \\
\hline Missed visits (dichotomous) & $0.994^{* *}$ & 1 & & & & \\
\hline Visit adherence (continuous, range: $0-1.0$ ) & $-0.852^{* *}$ & $-0.853^{* *}$ & 1 & & & \\
\hline $\begin{array}{l}\text { 4-month visit constancy (categorical, range: } \\
0-3 \text { ) }\end{array}$ & -0.027 & -0.050 & 0.074 & 1 & & \\
\hline 6-month gap (dichotomous) & 0.11 & 0.111 & -0.123 & -0.55 & 1 & \\
\hline HRSA HAB measure (dichotomous) & -0.133 & -0.131 & 0.125 & $0.182^{*}$ & 0.143 & 1 \\
\hline
\end{tabular}

HRSA HAB measure, the US Health Resources and Services Administration HIV/AIDS Bureau (HRSA HAB) measure; YALWH+T2DM, younger adults with HIV and T2DM ${ }^{*} p<0.05,{ }^{* *} p<0.01$

HAB measure had a low positive correlation with 4 -month visit constancy $(r=0.182, p<0.05)$ but was not correlated with other RIC measures. Moreover, unlike those in OALWH + T2DM and the entire sample, 4-month constancy in YALWH + T2DM was not related to the 6 -month visit gap.

\section{Health outcomes}

We investigated the relationship between RIC and CD4 count (Table 5). In OALWH + T2DM $(n=105)$, the only significant RIC measure that significantly predicted CD4 count was the number of missed visits $[\operatorname{Exp}(B)=11.277,95 \%$ confidence interval $(\mathrm{CI})$ (1.499, $84.843), p<0.05]$, indicating that having more missed visits predicted an 11-times greater chance of having a CD4 count $<500$ cells $/ \mu \mathrm{L}$ (Hosmer-Lemeshow Chi square,

Table 5 Associations between retention in care measures and CD4 cell count (<500 cells/ $\mu \mathrm{L})$ in PLWH + T2DM by age groups

\begin{tabular}{|c|c|c|c|c|c|c|c|c|c|}
\hline \multirow[t]{2}{*}{ Age group } & \multirow[t]{2}{*}{ Variables $^{a}$} & \multirow[t]{2}{*}{ B } & \multirow[t]{2}{*}{$S E$} & \multirow[t]{2}{*}{ Wald } & \multirow[t]{2}{*}{$d f$} & \multirow[t]{2}{*}{ Sig. } & \multirow[t]{2}{*}{$\operatorname{Exp}(B)$} & \multicolumn{2}{|l|}{$95 \% \mathrm{Cl}$} \\
\hline & & & & & & & & Lower & Upper \\
\hline \multirow[t]{9}{*}{ OALWH +T2DM $(n=105)$} & Missed visits (count) & 2.423 & 1.03 & 5.537 & 1 & $0.019^{*}$ & 11.277 & 1.499 & 84.843 \\
\hline & Missed visits (dichotomous) & 2.069 & 1.716 & 1.454 & 1 & 0.228 & 7.918 & 0.274 & 228.713 \\
\hline & Visit adherence & 5.582 & 5.733 & 0.948 & 1 & 0.33 & 265.714 & 0.004 & 20153270.43 \\
\hline & 4-month visit constancy & 0.685 & 0.566 & 1.464 & 1 & 0.226 & 1.983 & 0.654 & 6.014 \\
\hline & 6-month gap & 0.407 & 0.864 & 0.222 & 1 & 0.638 & 1.502 & 0.276 & 8.173 \\
\hline & HRSA HAB measure (retained) & 0.082 & 0.618 & 0.018 & 1 & 0.894 & 1.086 & 0.323 & 3.648 \\
\hline & Constant & -9.479 & 5.378 & 3.107 & 1 & 0.078 & 0 & & \\
\hline & \multicolumn{9}{|c|}{ Total Chi square $(6)=10.421, p>0.05$} \\
\hline & \multicolumn{9}{|c|}{ Hosmer-Lemeshow Chi square (3) $=0.89, p>0.05$} \\
\hline \multirow[t]{8}{*}{ YALWH +T2DM $(n=34)$} & Missed visits (count) & 10.804 & $16,337.328$ & 0 & 1 & 0.999 & $49,219.776$ & 0 & \\
\hline & Missed visits (dichotomous) & 30.158 & $62,616.205$ & 0 & 1 & 1 & $1.25186 \mathrm{E}+13$ & 0 & \\
\hline & Visit adherence & 4.645 & $235,894.115$ & 0 & 1 & 1 & 104.095 & 0 & \\
\hline & 4-month visit constancy & 0.185 & 1.645 & 0.013 & 1 & 0.911 & 1.203 & 0.048 & 30.251 \\
\hline & HRSA HAB measure (retained) & -0.849 & 1.465 & 0.336 & 1 & 0.562 & 0.428 & 0.024 & 7.56 \\
\hline & Constant & -35.14 & $180,003.341$ & 0 & 1 & 1 & 0 & & \\
\hline & \multicolumn{9}{|l|}{ Chi square $(6)=7.07, p>0.05$} \\
\hline & \multicolumn{9}{|c|}{ Hosmer-Lemeshow Chi square $(4)=2.29, p>0.05$} \\
\hline
\end{tabular}

HRSA HAB measure the US Health Resources and Services Administration HIV/AIDS Bureau (HRSA HAB) measure, PLWH+T2DM persons living with HIV and diabetes, all ages, OALWH + T2DM older adults with HIV and T2DM, YALWH+T2DM younger adults with HIV and T2DM

$* p<0.05$

a Step $1^{\mathrm{a}}$. variables: missed visits (count), missed visits (dichotomous), visit adherence, 4-month visit constancy, 6-month gap, HRSA HAB measure; Step $1^{\mathrm{b}}$. variables: missed visits (dichotomous), visit adherence, 4-month visit constancy, HRSA HAB measure 
Table 6 Standardized path coefficients by age groups

\begin{tabular}{|c|c|c|c|}
\hline Model & Path & Path coefficient & $p$ \\
\hline \multirow[t]{10}{*}{$\mathrm{OALWH}+\mathrm{T} 2 \mathrm{DM}$} & Standardized effects on CD4 cell count & & \\
\hline & Missed visits (count) & -0.46 & 0.001 \\
\hline & Attendance & -0.04 & 0.73 \\
\hline & Visit adherence & -0.43 & 0.002 \\
\hline & 4-month visit constancy & 0.22 & 0.040 \\
\hline & Standardized effects on HbAlc level & & \\
\hline & Missed visits (count) & -0.02 & 0.89 \\
\hline & Attendance & -0.03 & 0.78 \\
\hline & Visit adherence & 0.02 & 0.90 \\
\hline & 4-month visit constancy & 0.024 & 0.84 \\
\hline \multirow{10}{*}{ YALWH + T2DM } & Standardized effects on CD4 cell count & & \\
\hline & Missed visits (count) & -0.54 & 0.001 \\
\hline & Attendance & -0.20 & 0.26 \\
\hline & Visit adherence & -0.50 & 0.01 \\
\hline & 4-month visit constancy & -0.02 & 0.91 \\
\hline & Standardized effects on $\mathrm{HbA} 1 \mathrm{c}$ level & & \\
\hline & Missed visits (count) & 0.72 & 0.001 \\
\hline & Attendance & 0.32 & 0.04 \\
\hline & Visit adherence & 0.35 & 0.03 \\
\hline & 4-month visit constancy & -0.17 & 0.23 \\
\hline
\end{tabular}

PLWH + T2DM persons living with HIV and diabetes, all ages, OALWH + T2DM older adults with HIV and T2DM, YALWH + T2DM younger adults with HIV and T2DM

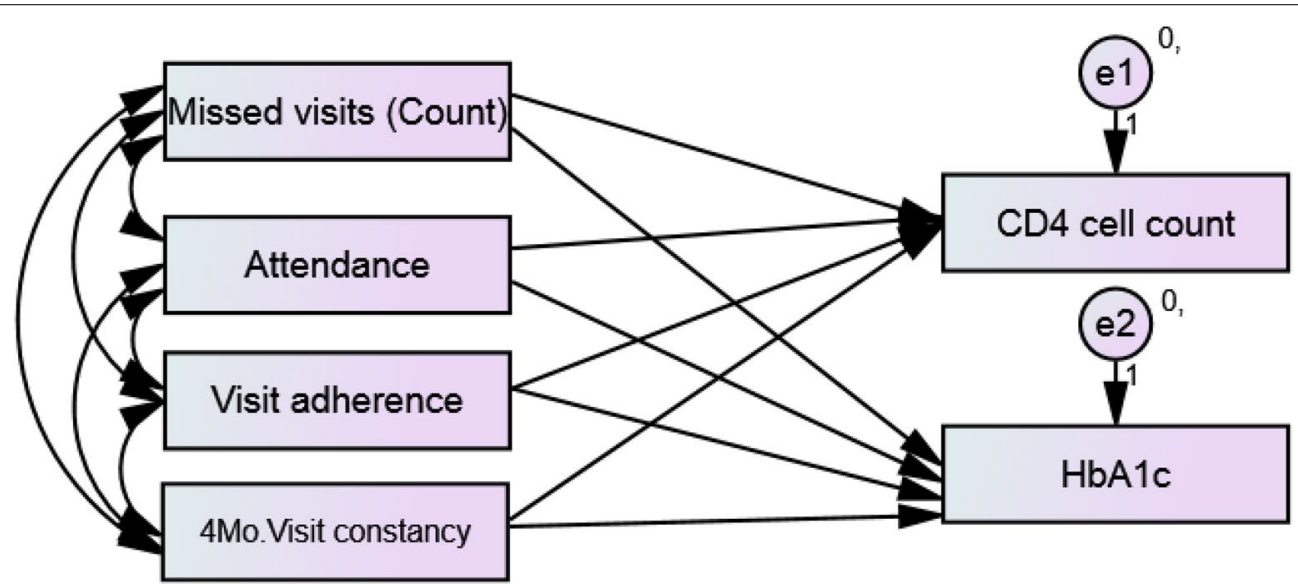

Fig. 1 Hypothesized path model in PLWH+T2DM. el error 1, e2 error 2, 4Mo. 4 month

$p<0.05)$. However, no RIC measures predicted CD4 count in YALWH + T2DM $(n=34)$.

\section{RIC and disease control paths}

Path analysis was conducted to assess relationships among RIC measures [missed visit (count), attendance, visit adherence, 4-month visit constancy, and disease control (CD4 cell count, HbA1c level)] in OALWH + T2DM and YALWH + T2DM (Table 6). Figure 1 presents the hypothesized path model for PLWH + T2DM, and Figs. 2 and 3 show the hypothesized models with path coefficients for OALWH + T2DM and YALWH+T2DM, respectively. Path analysis for OALWH + T2DM showed three significant hypothesized paths [missed visits (count), visit adherence, 4-month visit constancy] in CD4 count $(p<0.05)$ (Table 6). The model fit was good. Missed visits (count), visit adherence, and 4-month visit constancy were significantly related to CD4 count $(p<0.05)$. 


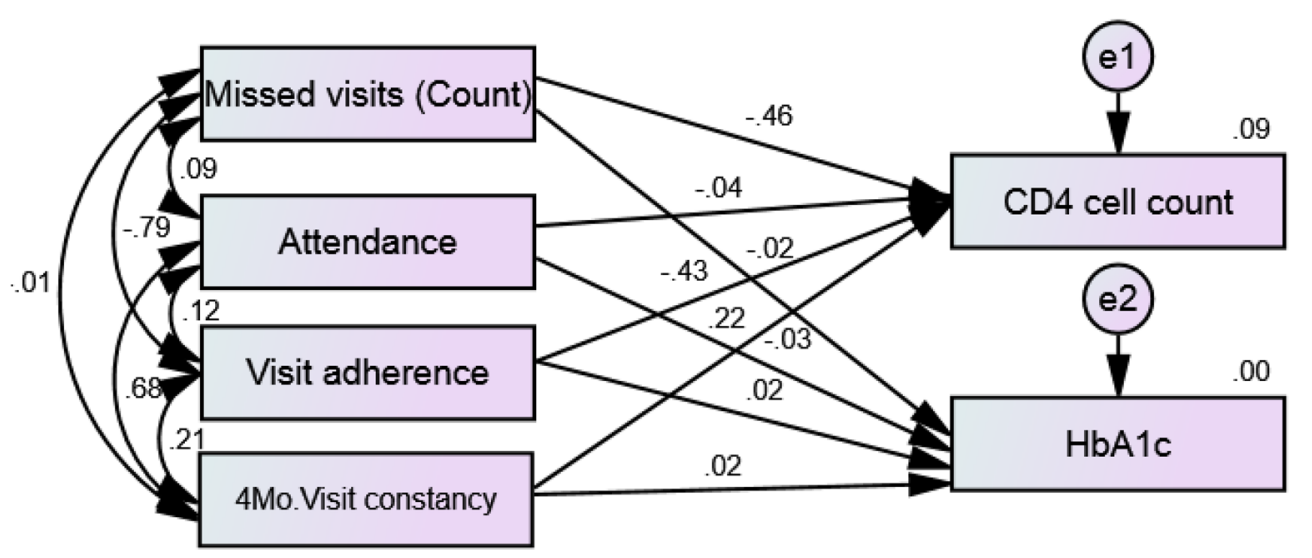

Chi-square $(\mathrm{df})=0.066(2)$

Probability $(\mathrm{P})=0.968$

Likelihood ratio $($ Chi-square/df $)=0.033$

$\mathrm{NFI}=1.00$

RMSEA $=0.00$

$\mathrm{CFI}=1.00$

$\mathrm{TLI}=1.03$

Fig. 2 Hypothesized model with path coefficients in OALWH+T2DM. CFI comparative fit index, e1 error 1, e2 error 2, 4Mo. 4 month, NFI normed fit index, RMSEA root mean square error of approximation, TLITucker-Lewis Index

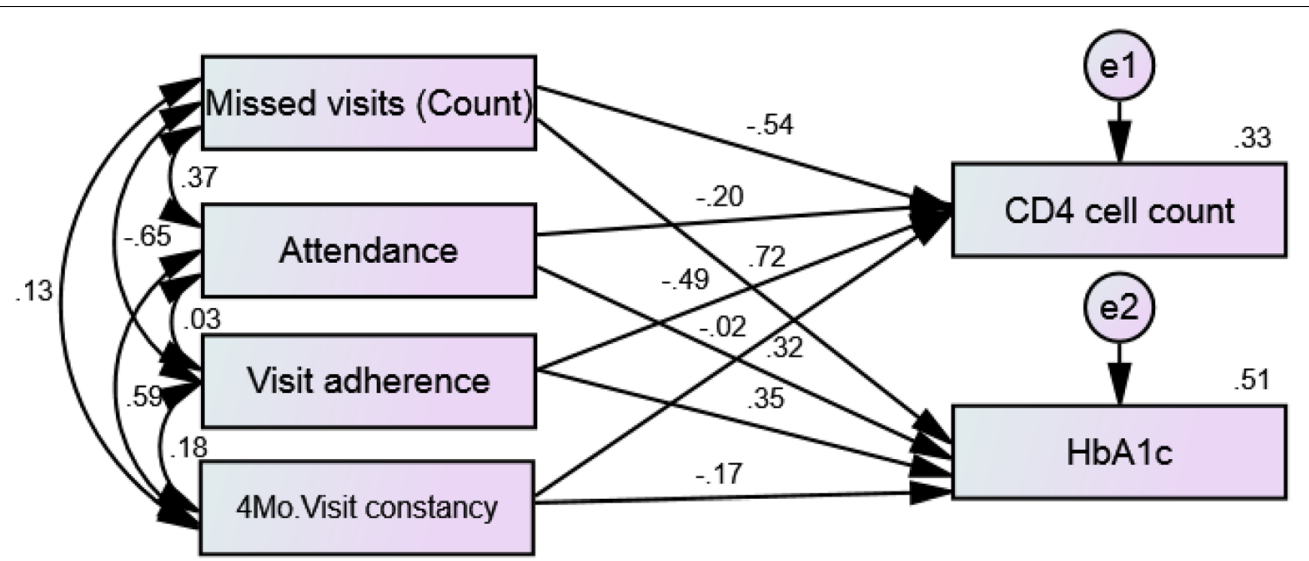

Chi-square $(\mathrm{df})=0.066(2)$

Probability $(\mathrm{P})=0.968$

Likelihood ratio $($ Chi-square/df $)=0.033$

$\mathrm{NFI}=1.00$

RMSEA $=0.00$

$\mathrm{CFI}=1.00$

$\mathrm{TLI}=1.03$

Fig. 3 Hypothesized model with path coefficients in YALWH+T2DM. CFI comparative fit index, el error 1, e2 error 2, 4Mo. 4 month, NFI normed fit index, RMSEA root mean square error of approximation, TLITucker-Lewis Index 
However, there were no significant relationships among missed visits (count), attendance, visit adherence, 4-month visit constancy, and HbA1c level $\left(\chi^{2}(2)=0.066\right.$, $p=0.968, \quad \mathrm{NFI}=1.0, \quad \mathrm{RMSEA}=0.00, \quad \mathrm{CFI}=1.00$, TLI $=1.03)(n=546)$. Visit adherence was significantly related to missed visits (count) and 4-month visit constancy $(p<0.05)$. Path analysis showed some differences between YALWH + T2DM and OALWH + T2DM. In YALWH + T2DM $\left(\chi^{2}[2]=0.066, p=0.968, \quad \mathrm{NFI}=1.0\right.$, RMSEA $=0.00$, CFI $=1.00, \mathrm{TLI}=1.03, n=226$; Table 7), missed visits (count) and visit adherence were significantly related to CD4 cell count $(p<0.05)$. Missed visits (count), attendance, and visit adherence were significantly related to HbA1c level $(p<0.05)$. Moreover, missed visits (count) were significantly related to visit attendance and adherence $(p<0.05)$. The model fit was good.

\section{Discussion}

The patients in this study had few missed visits, and the large majority (84\%) had not missed any visits in the year. Moreover, $72 \%$ had attended visits in two or three 4-month periods in a year, and $94 \%$ did not have a gap of 6 months between appointments. More than twothirds had attended two visits in the year with at least 90-day intervals. This is higher than the national average reported by the Centers for Disease Control and Prevention, which reported that $57.7 \%$ of their sample $(\mathrm{N}=358,151)$ was retained in care [32]. Almost twothirds were in poor T2DM control and more than twothirds of the sample had good control of HIV. Thus, the sample was relatively healthy. This could be attributed to the CNICS clinic. The clinics in this consortium offer a variety of services and opportunities to be a part of research studies. Being connected to a larger HIV community may be protective.

In this study, we examined the differences in RIC measures among PLWH in two age groups. The two different age groups had largely similar characteristics. The difference in ethnicity between the age groups may be accounted for by the shorter life expectancy of African American PLWH [33]. OALWH + T2DM had significantly lower visit attendance than YALWH + T2DM $(p=0.01)$. This result could possibly be due to insufficient access of OALWH + T2DM to clinic services, perhaps because older adults are more likely to have inadequate social support than younger adults (e.g., they may live alone or lack health insurance) [18]. Thus, some OALWH simply might not visit the clinic.

We also assessed the usefulness of six RIC measures for the sample and by age group. Spearman's correlations revealed differences in measures in PLWH + T2DM. Four-month visit constancy was not correlated with other RIC measures. This might be because 4-month visit constancy, unlike other measures, was assessed in 12 months and divided into 4-month segments. PLWH + T2DM might not have visited the clinic in each 4-month segment because they are not frequently scheduled appointments if their HIV status is under control. However, Mugavero et al. [26] who did not consider PLWH and additional comorbidities, found that all RIC measures, including the 4-month visit constancy, had significant correlations in PLWH. Perhaps, 4-month visit constancy may not be an appropriate measure for PLWH with comorbidities.

Logistic regression showed that only missed visits (count) significantly predicted CD4 count in OALWH + T2DM; other measures were not significantly associated with CD4 count. We expected stronger correlations, as reported by Mugavero et al. [26]. However, Mugavero's sample population was younger with more female and African American individuals. The major difference was that all patients in our sample were diagnosed with T2DM, which may change RIC if more appointments are necessary to manage each condition. This disconnect between missed appointments and higher CD4 count, may mean that patients with track records of strong immune systems did not feel it necessary to make as many appointments, or were able to get refills on their medication without an in-person visit. Changes in the increased availability of tele-health visits may see improvements in the amount of missed visits. Further research is needed in this area.

Lastly, this study performed path analysis for factors affecting the CD4 count and HbA1c levels in PLWH + T2DM divided into two age groups. Missed visits affected CD4 counts regardless of age, suggesting that RIC is important in HIV disease control $(p<0.05)$. Most

Table 7 Model fit comparisons by age group

\begin{tabular}{lllllll}
\hline Age group & $\mathbf{X}^{\mathbf{2}}$ & $\boldsymbol{d f}$ & $\boldsymbol{P}$ & NFI & RMSEA & CFI \\
\hline OALWH+T2DM & 0.066 & 2 & 0.968 & 1.0 & 0.00 & 1.00 \\
YALWH+T2DM & 0.066 & 2 & 0.968 & 1.0 & 0.00 & 1.03 \\
\hline
\end{tabular}

For all models, $p<0.05$

CFI comparative fit index, NFI normed fit index, TL/Tucker-Lewis Index, RMSEA root mean square error of approximation, PLWH +T2DM persons living with HIV and diabetes, all ages, OALWH + T2DM older adults with HIV and T2DM, YALWH + T2DM younger adults with HIV and T2DM 
RIC measures were significantly correlated with HbA1c level in YALWH + T2DM but not in OALWH + T2DM $(p<0.05)$.

These results are partially consistent with those of Zuniga et al. [34] who found that OALWH have better control of T2DM than YALWH. However, one main area of T2DM control in PLWH, i.e., RIC in older adults was not significantly correlated with the HbA1c level. This may be because T2DM is a disease that emphasizes selfmanagement-daily glucose monitoring, dietary management [35], and medication adherence [36] - and perhaps because OALWH + T2DM are more motivated or able to manage T2DM than YALWH + T2DM [36].

An important contribution of this study is the investigation of the interrelationships of RIC measures. We found that RIC can positively affect comorbidities and HIV infection, at least in the older population.

\section{Limitations}

Although the CNICS dataset represents a large, nationally representative sample of PLWH, this study has several limitations. The sample population comprised only those who visited the eight clinics in the CNICS network, which might limit generalizability to other populations. The study did not include those who might have died in calendar year 2015; thus, the results could be biased toward healthier patients. CD4 was selected over viral load because of there was less missing data with CD4. Since there were missing data on CD4 count and HbA1c levels that could have led to bias in the statistical analysis. Future studies should aim to include more complete samples of CD4 count and HbA1c levels in PLWH + T2DM.

\section{Conclusions}

We conducted a comparative analysis on RIC in PLWH + T2DM who are part of a large national cohort study in one calendar year. RIC measures predicted CD4 count regardless of age. Nonetheless, the HbA1c level was significantly affected in YALWH + T2DM. This is the first study to consider PLWH + T2DM in terms of age groups. Even though our study contained a limited sample of CD4 count and HbA1c level data, our results will guide research on the relationships between RIC and CD4 counts and HbA1c levels in PLWH+T2DM in the future.

\footnotetext{
Abbreviations

ART: Antiretroviral therapy; CFAR: Center for AIDS Research; CFI: Comparative fit index; CNICS: Center for AIDS Research Network of Integrated Clinical Systems; NFI: Normed fit index; OALWH: Older adults living with HIV; PLWH: Persons living with HIV; RIC: Retention in care; RMSEA: Root mean square error of approximation; TLI:Tucker-Lewis Index; YALWH: Younger adults living with HIV.
}

\section{Acknowledgements}

The Center for AIDS Research (CFAR) Network of Integrated Clinical Systems (CNICS) is an National Institutes of Health (NIH)-funded program (R24 Al067039) made possible by the National Institute of Allergy and Infectious Diseases and National Heart, Lung, and Blood Institute. The CFAR sites involved in the CNICS include the University of Alabama at Birmingham (P30 AI027767); University of Washington (P30 Al027757); University of California, San Diego (P30 Al036214); University of California, San Francisco (P30 Al027763); Case Western Reserve University (P30 Al036219); Johns Hopkins University (P30 Al094189, U01 DA036935); Fenway Health/Harvard (P30 Al060354); and University of North Carolina at Chapel Hill (P30 Al50410). Editorial support with manuscript development was provided by the Cain Center for Nursing Research and Center for Transdisciplinary Collaborative Research in Selfmanagement Science (P30, NR015335) at The University of Texas at Austin School of Nursing. This manuscript is adapted from a dissertation. This work was supported by the National Research Foundation of Korea (NRF) grant funded by the Korea government (MSIT) (No. 202000000001280). This work was supported by the research fund of Hanyang University (HY-2019).

\section{Authors' contributions}

The following statements should be used "conceptualization, methodology, formal analysis, investigation, resources, data curation, writing —original draft preparation, writing, data management, JP; review and editing, supervision, project administration, JAZ; review, editing AAG, and data management JL. All authors read and approved the final manuscript.

\section{Funding}

This work was supported by the National Research Foundation of Korea (NRF) grant funded by the Korea government (MSIT) (No. 202000000001280). This work was supported by the research fund of Hanyang University (HY-2019).

\section{Availability of data and materials}

The data that support the findings of this study are available from the Center for AIDS Research (CFAR) Network of Integrated Clinical Systems (CNICS), but restrictions apply to the availability of these data, which were used under license for the current study, and so are not publicly available. Data are however available from the authors upon reasonable request and with permission of CNICS.

\section{Ethics approval and consent to participate}

This study was exempted from by the Institutional Review Board (IRB) at the University of Texas at Austin because it used de-identified data.

\section{Consent for publication \\ Not applicable.}

\section{Competing interests}

The authors declare that they have no competing interests.

\section{Author details \\ ${ }^{1}$ School of Nursing, The University of Texas at Austin, Austin, TX, USA. ${ }^{2}$ Division of Community Engagement and Health Equity, Department of Population Health, Dell Medical School, The University of Texas at Austin, Austin, TX, USA. ${ }^{3}$ Electrical and Computer Engineering, The University of Texas at Austin, Austin, TX, USA. ${ }^{4}$ School of Nursing, Hanyang University, 222 Wangsimni-ro, Sageun-dong, Seongdong-gu, Seoul, South Korea.}

Received: 28 January 2020 Accepted: 15 May 2020

Published online: 29 May 2020

\section{References}

1. Centers for Disease Control and Prevention. HIV/AIDS: Statistics Overview; 2017 http://www.cdc.gov/hiv/statistics/basics. Accessed 2 Aug 2019.

2. Samji H, Cescon A, Hogg RS, Modur SP, Althoff KN, Buchacz K, et al. Closing the gap: increases in life expectancy among treated HIV-positive individuals in the United States and Canada. PLoS ONE. 2013;8:e81355. https://doi.org/10.1371/journal.pone.0081355.

3. Antiretroviral Therapy Cohort Collaboration. Life expectancy of individuals on combination antiretroviral therapy in high-income countries: a 
collaborative analysis of 14 cohort studies. Lancet. 2008;372:293-9. https ://doi.org/10.1016/S0140-6736(08)61113-7.

4. Rolls S, Denneny E, Marcus R, O'Connell R. Tackling cardiovascular comorbidities in HIV-positive patients: who, how and where? J Int AIDS Soc. 2014;17:19725. https://doi.org/10.7448/IAS.17.4.19725.

5. Brown TT, Cole SR, Li X, Kingsley LA, Palella FJ, Riddler SA, et al. Antiretroviral therapy and the prevalence and incidence of diabetes mellitus in the multicenter AIDS cohort study. Arch Intern Med. 2005;165:1179-84. https ://doi.org/10.1001/archinte.165.10.1179.

6. Butt AA, McGinnis K, Rodriguez-Barradas MC, Crystal S, Simberkoff M, Goetz MB, et al. HIV infection and the risk of diabetes mellitus. AIDS. 2009;23:1227-34. https://doi.org/10.1097/QAD.0b013e32832bd7af.

7. Medapalli RK, Parikh CR, Gordon K, Brown ST, Butt AA, Gibert CL, et al. Comorbid diabetes and the risk of progressive chronic kidney disease in HIV-infected adults: data from the veterans aging cohort study. J Acquir Immune Defic Syndr. 2012;60:393-9. https://doi.org/10.1097/QAl.0b013 e31825b70d9.

8. Vance DE, Mugavero M, Willig J, Raper JL, Saag MS. Aging with HIV: a cross-sectional study of comorbidity prevalence and clinical characteristics across decades of life. J Assoc Nurses AIDS Care. 2011;22:17-25. https ://doi.org/10.1016/j.jana.2010.04.002.

9. Brown TT, Tassiopoulos K, Bosch RJ, Shikuma C, McComsey GA. Association between systemic inflammation and incident diabetes in HIVinfected patients after initiation of antiretroviral therapy. Diabetes Care. 2010;33:2244-9. https://doi.org/10.2337/dc10-0633.

10. De Wit S, Sabin CA, Weber R, Worm SW, Reiss P, Cazanave C, et al. Incidence and risk factors for new-onset diabetes in HIV-infected patients: the data collection on adverse events of anti-HIV drugs (D:A:D) study. Diabetes Care. 2008;31:1224-9. https://doi.org/10.2337/dc07-2013.

11. Huang ES, Laiteerapong N, Liu JY, John PM, Moffet HH, Karter AJ. Rates of complications and mortality in older patients with diabetes mellitus: the diabetes and aging study. JAMA Intern Med. 2014;174:251-8. https://doi. org/10.1001/jamainternmed.2013.12956.

12. Centers for Disease Control and Prevention. HIV/AIDS: HIV among people aged 50 and over; 2017. http://www.cdc.gov/hiv/group/age/olderameri cans/. Accessed 20 Oct 2018.

13. Effros RB, Fletcher CV, Gebo K, Halter JB, Hazzard WR, Horne FM, et al. Workshop on HIV infection and aging: what is known and future research directions. Clin Infect Dis. 2008;47:542-53. https://doi.org/10.1086/59015 0.

14. Emlet CA. A comparison of HIV stigma and disclosure patterns between older and younger adults living with HIV/AIDS. AIDS Patient Care STDs. 2006;20:350-8. https://doi.org/10.1089/apc.2006.20.350.

15. Legarth RA, Ahlström MG, Kronborg G, Larsen CS, Pedersen C, Pedersen G, et al. Long-term mortality in HIV-infected individuals 50 years or older: a nationwide, population-based cohort study. J Acquir Immune Defic Syndr. 2016;71:213-8. https://doi.org/10.1097/QAl.00000000000000825.

16. Savasta AM. HIV: associated transmission risks in older adults—an integrative review of the literature. J Assoc Nurses AIDS Care. 2004;15:50-9. https ://doi.org/10.1177/1055329003252051.

17. Webel AR, Longenecker CT, Gripshover B, Hanson JE, Schmotzer BJ, Salata RA. Age, stress, and isolation in older adults living with HIV. AIDS Care. 2014:26:523-31. https://doi.org/10.1080/09540121.2013.845288.

18. Emlet CA, Farkas KJ. Correlates of service utilization among midlife and older adults with HIV/AIDS: the role of age in the equation. J Aging Health. 2002;14:315-35. https://doi.org/10.1177/08964302014003001.

19. Negin J, Martiniuk A, Cumming RG, Naidoo N, Phaswana-Mafuya N, Madurai L, et al. Prevalence of HIV and chronic comorbidities among older adults. AIDS. 2012;26:S55-63. https://doi.org/10.1097/QAD.0b013 e3283558459.

20. Geng EH, Nash D, Kambugu A, Zhang Y, Braitstein P, Christopoulos KA, et al. Retention in care among HIV-infected patients in resource-limited settings: emerging insights and new directions. Curr HIV/AIDS Rep. 2010;7:234-44. https://doi.org/10.1007/s11904-010-0061-5.
21. Mugavero MJ, Amico KR, Westfall AO, Crane HM, Zinski A, Willig JH, et al. Early retention in HIV care and viral load suppression: implications for a test and treat approach to HIV prevention. J Acquir Immune Defic Syndr. 2012;59:86-93. https://doi.org/10.1097/QAl.0b013e318236f7d2.

22. Attia S, Egger M, Müller M, Zwahlen M, Low N. Sexual transmission of HIV according to viral load and antiretroviral therapy: systematic review and meta-analysis. AIDS. 2009;23:1397-404. https://doi.org/10.1097/ QAD.0b013e32832b7dca.

23. Thompson MA, Mugavero MJ, Amico KR, Cargill VA, Chang LW, Gross $R$, et al. Guidelines for improving entry into and retention in care and antiretroviral adherence for persons with HIV: evidence-based recommendations from an International Association of Physicians in AIDS Care panel. Ann Intern Med. 2012;156:817-33. https://doi.org/10.7326/00034819-156-11-201206050-00419.

24. Cohen SM, Van Handel MM, Branson BM, Blair JM, Hall HI, Hu X, et al. Vital signs: HIV prevention through care and treatment—United States. Morb Mortal Wkly Rep. 2011;60:1618-23.

25. Giordano TP, Gifford AL, White ACJ, Almazor MES, Rabeneck L, Hartman $C$, et al. Retention in care: a challenge to survival with HIV infection. Clin Infect Dis. 2007:44:1493-9. https://doi.org/10.1086/516778.

26. Mugavero MJ, Westfall AO, Zinski A, Davila J, Drainoni ML, Gardner LI, et al. Measuring retention in HIV care: the elusive gold standard. J Acquir Immune Defic Syndr. 2012;61:574-80. https://doi.org/10.1097/QAl.0b013 e318273762f.

27. US Department of Veterans Affairs. HIV/AIDS: CD4 count (or T-cell count); 2016. https://www.hiv.va.gov/patient/diagnosis/labs-CD4-count.asp. Accessed 20 Oct 2018.

28. Nasir NM, Thevarajah M, Yean CY. Hemoglobin variants detected by hemoglobin $\mathrm{A} 1 \mathrm{c}(\mathrm{HbA} 1 \mathrm{c})$ analysis and the effects on $\mathrm{HbA} 1 \mathrm{c}$ measurements. Int J Diabetes Dev Ctries. 2010;30:86-90. https://doi. org/10.4103/0973-3930.62598.

29. Cagliero E, Levina EV, Nathan DM. Immediate feedback of HbA1c levels improves glycemic control in type 1 and insulin-treated type 2 diabetic patients. Diabetes Care. 1999;22:1785-9. https://doi.org/10.2337/diaca re.22.11.1785

30. Eckhardt BJ, Holzman RS, Kwan CK, Baghdadi J, Aberg JA. Glycated hemoglobin $A_{1 C}$ as screening for diabetes mellitus in HIV-infected individuals. AIDS Patient Care STDs. 2012;26:197-201. https://doi.org/10.1089/ apc.2011.0379.

31. Tien PC, Schneider MF, Cox C, Karim R, Cohen M, Sharma A, et al. Association of HIV infection with incident diabetes mellitus: impact of using hemoglobin A1C as a criterion for diabetes. J Acquir Immune Defic Syndr. 2012;61:334-40. https://doi.org/10.1097/QAl.0b013e31826bfc32.

32. IBM Corp. IBM SPSS Statistics 23.0. Armonk: IBM Corp; 2015.

33. Arbuckle JL. Amos (version 23.0) [Computer Program]. SPSS, Chicago; 2014.

34. Singh S, Mitsch A, Wu B. HIV care outcomes among men who have sex with men with diagnosed IV infection—United States, 2015. Morb Mortal Wkly Rep. 2017;66:969-74.

35. Harrison KM, Song R, Zhang X. Life expectancy after HIV diagnosis based on national HIV surveillance data from 25 states, United States. J Acquir Immune Defic Syndr. 2010;53:124-30. https://doi.org/10.1097/QAl.0b013 e3181b563e7.

36. Zuniga J, Nguyen ML, Holstad M. Predictors of dual control of HIV and diabetes. AIDS Care. 2016;28:1124-7. https://doi.org/10.1080/09540 121.2016.1139667.

\section{Publisher's Note}

Springer Nature remains neutral with regard to jurisdictional claims in published maps and institutional affiliations. 